\title{
Borate Glasses for Luminescence Applications - Potential Materials for White LEDs and Laser Sources
}

\author{
K. Mariselvam ${ }^{1,2}$, R. Arun Kumar, ${ }^{1,2, *}$ \\ ${ }^{1}$ GRD Centre for Materials Research, PSG College of Technology, India \\ ${ }^{2}$ Department of Physics, PSG College of Technology, India
}

Copyright $\bigcirc 2016$ by authors, all rights reserved. Authors agree that this article remains permanently open access under the terms of the Creative Commons Attribution License 4.0 International License

\begin{abstract}
The development of borate based glasses for luminescence applications are reviewed. The present work covers various aspects including the search and synthesis of new materials, the formation of glasses, the structural and optical properties of absorption, excitation and luminescence on the prepared glasses, Information on the Judd-Ofelt intensity parameters as calculated from absorption spectra and the studies on the emission properties. CIE colour coordinates are helpful to analyze the potentiality of the glasses for white light emission. We aim to provide a comprehensive review of borate glass materials for LED and screen display applications etc.
\end{abstract}

Keywords Luminescence, Judd-Ofelt Parameters, CIE Colour Chromaticity, Stimulated Emission Cross Section, White LEDs

\section{Introduction}

Light is a form of energy. To produce light, another form of energy must be supplied. There are two common ways to produce light, incandescence and luminescence. Incandescence is generating light from heat energy. The phenomena which involve absorption of light energy and subsequent emission of light are come under the term 'luminescence'. Luminescence is the emission of light by a substance. It can be caused by electrical energy, chemical reactions, and subatomic motions. The term Luminescence was introduced in 1888 by Eilhard Wiedemann, in Latin 'lumen' means 'light'. The materials exhibiting this phenomenon are known as 'luminescent Materials' or 'phosphors' meaning 'light bearer' in Greek. The term phosphor was introduced in $17^{\text {th }}$ century by an Italian alchemist Vincentinus Casaiarolo of Balogona.

Phosphors are luminescent materials that emit light when excited by radiation, and are usually microcrystalline powders or thin-films designed to provide visible color emission. In an important group of luminescent materials, the transfer of energy to the luminescent center is brought about by the motion of electrons. Many oxides, sulphides, selenides, tellurides, arsenides, and phosphides are of this nature, and of these, zinc sulphide is been widely studied. It is frequently used as a luminescent material in cathode-ray tubes and electroluminescent lamps [1].

After few decades of research and development, thousands of phosphors have been prepared and some of them are identified and widely used in many applications. Recently, great strides have been made towards the production of white light-emitting diodes (LEDs) which have certain advantages such as high brightness, low power consumption, longevity, reliability and excellent low temperature performance. The LEDs are utilized in next generation solid illumination devices, backlighting of liquid crystal displays, automotive headlamps, advertising, general lighting, traffic signals, and camera flashes [2].

\subsection{Types of Luminescence}

The following are the different types of luminescence.

\subsubsection{Chemi-luminescence}

This is light emitted during chemical reaction. Ex: Phosphorus, glow sticks or cyalume sticks.

\subsubsection{Bio-luminescence}

Emission is a result of biochemical reaction by living organism. Ex: Fireflies, comb jellies, angler fish.

\subsubsection{Electro-chemiluminescence}

A result of an electrochemical reaction: Ex: Light-emitting electrochemical cell, image analysis.

\subsubsection{Electro-luminescence}

A result of an electric current passed through a substance. Ex: LED, OLED, PLED, AMOLED, LASER, electroluminescent wires.

\subsubsection{Tribo-luminescence}

Generated when bonds in a material are broken that 
material is crashed: Ex: Diamond.

\subsubsection{Crystalo-luminescence}

Crystallo-luminescence is a type of Luminescence generated during crystallization and it is used to determine the critical size of the crystal nucleus. There is a theory that the light from Crystallo-luminescence emerges through the micro-fracture of growing crystallites. This effectively classifies Crystallo-luminescence as a type of Tribo-luminescence and a subtype of Electroluminescence.

\subsubsection{Mechano-luminescence}

A result of a mechanical action by a solid: Ex: Sensing and imaging devices.

\subsubsection{Fracto-luminescence}

Generated when bonds in certain crystals are broken by fractures: Ex: Quartz.

\subsubsection{Piezo-luminescence}

Produced by the action of pressure on certain solids: Ex: Actuating element in vehicles.

\subsubsection{Radio-luminescence}

A result of bombardment by ionizing radiation such as beta particles: Ex: Radio-luminescent paint is used for clock hands and instrument dial, enabling them to be read in dark.

\subsubsection{Thermo-luminescence}

The re-emission of absorbed energy when a substance is heated: Ex: Dating some archeological artifacts.

\subsubsection{Cryo-luminescence}

The emission of light when an object is cooled: Ex: Ratio metric thermometer, solar cell.

\subsubsection{Seismo-luminescence}

Seismoluminescence is a reference to Earthquake Lights, rare glowing phenomena that are occasionally seen before or during seismic event. Ex: Lightning

\subsubsection{Photoluminescence}

Photoluminescence (PL) processes can be classified by various parameters such as the energy of the exciting photon with respect to the emission. Optical excitation by absorption of a photon leads to a major class of technically important luminescent species which fluorescence or phosphorescence. PL is simple, versatile, and non-destructive in nature. PL investigations can be used to characterize a variety of material parameters. PL spectroscopy provides electrical (as opposed to mechanical) characterization, and it is a selective and extremely sensitive probe of discrete electronic states. Features of the emission spectrum can be used to identify surface, interface, and impurity levels and to gauge alloy disorder and interface roughness. The advantages of PL analysis are the simplicity of optical measurements and the power to probe fundamental electronic properties [4].

\section{Fluorescence}

In general, fluorescence is a "fast" process, and photoluminescence in which the emitted photons are of lower energy than those absorbed. Afterglow is observed independent of the excitation intensity and of temperature, with lifetime less than $10^{-8}$ seconds. Ex: Fluorescent lamps.

\section{Phosphorescence}

In general, phosphorescence is a "slow" process, and fluorescence slightly delayed after initial absorption of radiation. Afterglow is observed dependent of the excitation intensity and strongly temperature dependent, with life time more than $10^{-8}$ seconds. Ex: Light sources, multi colour displays.

\subsection{Excitation and Emission Spectra}

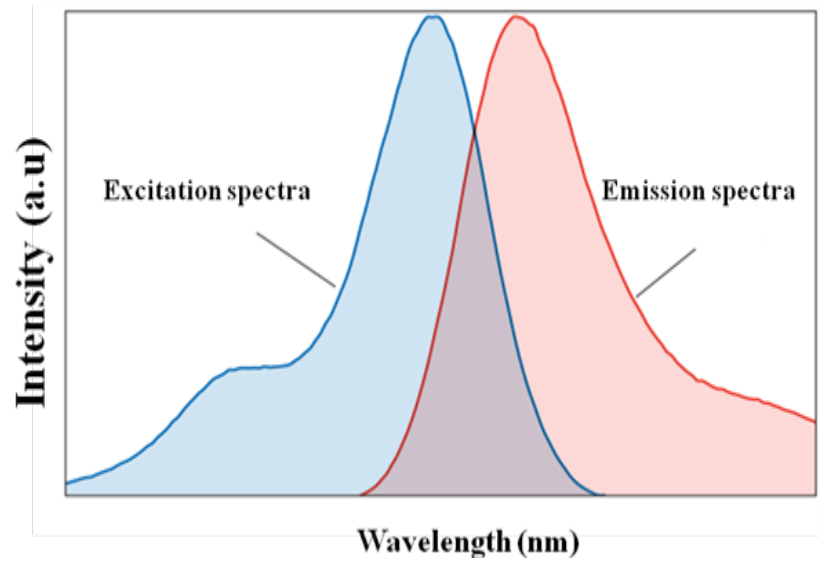

Figure 1. Excitation and Emission Spectra

Table 1. Visible range of the spectrum from the electromagnetic radiation

\begin{tabular}{|c|c|}
\hline Visible spectrum & Visible range \\
\hline Violet and indigo & $400-450 \mathrm{~nm}$ \\
\hline Blue and aqua & $450-500 \mathrm{~nm}$ \\
\hline Green & $500-570 \mathrm{~nm}$ \\
\hline Yellow and orange & $570-610 \mathrm{~nm}$ \\
\hline Red & 610 to approximately $750 \mathrm{~nm}$ \\
\hline
\end{tabular}

Figure (1) shows a typical spectrum of the excitation and emission of a fluorochrome. These spectra are generated by an instrument called a spectrofluorometer. The colour spectra are described quantitatively by the wavelength of emitted light. The colours of the visible spectrum can be broken up into the approximate wavelength ranges as shown in table 1 [3]. On the short-wavelength end of the visible spectrum the near-ultraviolet (near-UV) band from 320 to $400 \mathrm{~nm}$ and on the long-wavelength end is the near infrared (near-IR) band from 750 to approximately $2500 \mathrm{~nm}$ were tabulated in Table 1. The broad band of light from 320 to $2500 \mathrm{~nm}$ marks the limits of transparency of prepared glasses and this is the band most often used in fluorescence 
microscopy [4].

\section{Experimental Methods}

The luminescent materials are synthesized by solid state reaction technique at high temperatures in ambient conditions. All the precursor chemicals taken in appropriate proportions are crushed in an agate mortar until a homogeneous mixture was obtained. The homogeneous mixture is taken is a suitable crucible like platinum or silica or aluminium. The crucible is then heated at high temperature around $\left(1200{ }^{\circ} \mathrm{C}\right)$ in an electric furnace for a desired period, and cooled rapidly to obtain homogeneously mixed melt. Such melt is then quenched suddenly on a pre-heated brass mould and pressed quickly by another brass mould. The glass samples thus prepared were annealed for around 3 to 4 hours for the removal of thermal stress acquired by glass matrix during quenching.

\section{Common analytical Techniques}

The prepared and well polished glasses are subjected to physical properties analysis, structural analysis and optical analysis. The following properties are studied.

\subsection{Physical Properties}

Density

The densities of the prepared glasses are measured at room temperature with the help of immersion in a liquid by using the Archimedes principle. The examples of liquids water, xylene and monobromonaphthalene etc.

Refractive Index

Refractive indices for the prepared glasses were measured by using Brewster's law with some sources. Ex: laser (He-Ne) $650 \mathrm{~nm}$, sodium vapour lamp (589 nm). Refractive index is a critical parameter in the control of mode profile and can affect the performance of an optical amplifier. The higher refractive indices observed for these glasses make them as promising material for optical fiber with more numerical aperture values.

The following terms are some physical properties to study glassy material for effective luminescence applications.

Atomic volume: atomic weight / $\operatorname{density}\left(\mathrm{g} / \mathrm{cm}^{3} /\right.$ atom $)$

$$
\text { Dielectric constant }{ }^{(\varepsilon)}: \quad \varepsilon=n^{2}
$$

Where $\mathrm{n}$ is the refractive index of the glass.

$$
\begin{aligned}
& \text { Reflection losses (R): R }(\%)=\left(\frac{n-1}{n+1}\right)^{2} \times 100 \\
& \text { Molar Refractivity }\left(\mathbf{R}_{\mu}\right): R_{\mu}=\left(\frac{n^{2}-1}{n^{2}+2}\right) \frac{M}{\rho}
\end{aligned}
$$

Where $\mathrm{M}$ is the average molecular weight of the glass and $\rho$ is the density.

Inter atomic distance $\left(\mathrm{r}_{\mathrm{i}}\right)$ :

$$
\begin{gathered}
\qquad\left(r_{i}\right)(A)=\left(\frac{1}{N}\right)^{\frac{1}{3}} \\
\text { Polaran radius }\left(\mathbf{r}_{\mathrm{p}}\right): \mathrm{r}_{\mathrm{p}}(A)=\frac{1}{2}\left(\frac{\pi}{6 N}\right)^{\frac{1}{3}} \\
\text { Field strength }(\mathbf{F}): \quad F=\frac{1}{4 \pi \varepsilon_{0}} \frac{Z}{r_{p}^{2}}
\end{gathered}
$$

Where $\mathrm{Z}$ is the charge of the ion; $\mathrm{Z}=3 \mathrm{e}$; e is the electron charge, $\varepsilon_{0}$ is the permittivity of free space $r_{p}$ is the distance between two points.

\section{Numerical Aperture (NA)}

When the glass under investigation is used as the core of the optical fiber;

$$
N A=n \sqrt{2 \Delta}
$$

Where $\Delta$ is the fractional RI change:

$$
\Delta=\frac{n-n_{\text {cladding }}}{n}
$$

Electric susceptibility $(\chi): \chi=\frac{n^{2}-1}{4 \pi}$

\subsection{Structural Analysis}

Powder X-Ray Diffraction Analysis

Powder X-Ray Diffraction method is a standard technique to confirm the amorphous nature of the glassy materials. The spectrum is usually recorded using an X-Ray diffractometer instrument and this spectrum does not show any sharp peaks but gives broad hump (noise) which is the characteristic feature of a glassy material that confirms its amorphous nature.

\section{FTIR Analysis}

Fourier transform infrared Spectroscopy (FTIR) is one of the most useful analytical methods to identify a functional group of chemical a composition. One advantage of IR over other analytical techniques is that spectra can be collected on small amounts of sample using $\mathrm{KBr}$ pellet technique.

\section{Raman Analysis}

Raman spectroscopy provides information about molecular vibrations that can be used for sample identification and quantization. 


\subsection{Optical Analysis}

\section{Absorption Spectral Analysis}

The absorption spectra for the prepared glasses are recorded in the 400-1000 $\mathrm{nm}$ wavelength range on a UV-Vis-NIR spectrophotometer and this instrument is equipped with a photomultiplier tube and a detector to acquire data in UV-Vis NIR region with a resolution $\leq 0.05 \mathrm{~nm}$. This method is very useful for the investigation of induced transitions and it gives information regarding band structure and energy gap of amorphous materials.

Nephelauxetic ratio $\left(\beta_{\mathrm{R}}\right)$ and bonding parameter $(\delta)$ :

The Nephelauxetic ratio $\left(\beta_{R}\right)$ is calculated by using the following expression

$$
\beta_{\mathrm{R}}=v_{\mathrm{c}} / v_{\mathrm{a}}
$$

Where $\beta_{R}$ is equal to the ratio between the observed wavenumber (in $\mathrm{cm}^{-1}$ ) of a particular transition of the RE ion under investigation and the wavenumber (in $\mathrm{cm}^{-1}$ ) for the corresponding transition of aquo-ion [5]. From the average values of $\beta$ (referred as $\bar{\beta}$ ) is used to estimate bonding parameter, $\delta$ using the relation

$$
\delta \%=\left[\frac{1-\bar{\beta}}{\bar{\beta}}\right] \times 100
$$

\section{Bandgap and Urbach's Energy Analysis}

Photons with energy greater than optical bandgap energy will get absorbed by the material and based on this principle the electronic band structure of an amorphous material can be studied through the absorption spectra. The bandgap in electronic band structure of solids generally refers to the energy difference between the top of the valence band and bottom of the conduction band in insulators and semiconductors. The bandgap is an important factor which determines the electrical conductivity of the solid material. Optical bandgap studies have proven to be an effective tool for investigating the electronic band structure of amorphous materials. The direct and indirect bandgap energy values can be determined through the optical absorption spectra at the fundamental absorption edge of the material. The absorption co-efficient $\alpha(v)$, near the absorption edge is calculated using the expression given below

$$
\alpha(v)=\left(\frac{1}{d}\right) \ln \left(\frac{I_{0}}{I_{t}}\right)
$$

Where $d$ is the thickness of the sample, $I_{0}$ and $I_{t}$ are the intensities of the incident and transmitted radiations respectively.

Urbach's energy expression given by Davis and Mott [6].

$$
\alpha(v)=\frac{B\left(h v-E_{o p t}\right)^{r}}{h v}
$$

The absorption coefficient $\alpha(v)$ as a function of photon energy $(h v)$ for the direct and indirect allowed transitions can be calculated, where $\mathrm{B}$ is the band tailing parameter, $\mathrm{E}_{\mathrm{opt}}$ is the optical bandgap energy, $h v$ is the incident photon energy and $r$ is the index number to determine the type of optical transition. The materials with larger Urbach energy would have greater tendency to convert weak bonds into defects. The decreasing Urbach's energy values indicate the presence of minimum number of defects in the prepared glasses. The Urbach's energy values vary inversely with optical bandgap values. Therefore, the lower Urbach energies indicate the lower extension of width of the tail of localized states into the forbidden band gap.

\section{Oscillator Strength and Judd-Ofelt Analysis}

The most difficult problem in rare earth spectroscopy is the measurement of the intensity of absorption band expressed in terms of oscillator strength, which gives the intensity of absorption band corresponding to a particular transition. The experimental oscillator strength $\left(f_{\text {exp }}\right)$ of electric dipole transition is defined as

$$
f_{\exp }=4.32 \times 10^{-9} \int_{v_{1}}^{v_{2}} \varepsilon(v) d v
$$

Where $\varepsilon(v)$ is the molar extinction coefficient corresponding to the energy $\left(v \mathrm{~cm}^{-1}\right)$ and $d v$ is the half bandwidth of the absorption band. According to JO theory, the oscillator strength $\left(f_{\text {cal }}\right)$ of an electric dipole-dipole absorption transition from initial state $|(S, L) J\rangle$ to final state $\left|\left(S^{\prime}, L^{\prime}\right) J^{\prime}\right\rangle$ depends on three $\Omega_{\lambda}(\lambda=2,4$ and 6$)$ as given by

$$
\begin{gathered}
f_{\text {cal }}\left[(S, L) J ;\left(S^{\prime}, L^{\prime}\right) J^{\prime}\right] \\
=\frac{8 \pi^{2} m c}{3 h \lambda(2 J+1)} \frac{\left(n^{2}+2\right)^{2}}{9 n} \sum_{\lambda=2,4,6} \Omega_{\lambda} \\
\left|<(S, L) J\left\|U^{(x)}\right\|\left(S^{\prime}, L^{\prime}\right) J^{\prime}>\right|^{2}
\end{gathered}
$$

Where $\mathrm{m}$ is the mass of the electron, $\lambda$ is the mean wavelength of the transition, $\mathrm{n}$ is the refractive index, $\Omega_{\lambda}$ the JO parameters and $\left\langle\left\|U^{(\Omega)}\right\|>\right.$ the doubly reduced matrix elements of unit tensor operator which are independent of the host matrix. These reduced matrix elements were taken from the reference [6] for the JO analysis. The oscillator strengths for the various transitions are calculated using the Equations. 15 and 16. A least square fitting approach is used for Eq. 16 to determine JO parameters, which gives the best fit between experimental and calculated oscillator strengths.

The Judd-Ofelt intensity parameters $\Omega_{\lambda}(\lambda=2,4$ and 6) measured through least squaring fitting method. $\Omega_{2}$ parameter is sensitive to both asymmetry and covalency at the rare earth sites and large value indicates a strong covalency of the metal-ligand bond in glasses. $\Omega_{4}$ parameter seems to be mainly dependent on the long range effects and related to the bulk properties of the glasses. $\Omega_{6}$ parameter indicates the rigidity of the medium in which the lanthanide ions are embedded. Although, the intensity parameters, $\Omega_{4}$, $\Omega_{6}$ need not depend on the local crystal field, they play a 
crucial role in defining the radiative properties such as transition probability, branching ratio and stimulated emission cross section of active ions. The ratio $\left(\Omega_{4} / \Omega_{6}\right)$ is known as the spectroscopic quality factor $(\chi)$.

\section{Emission Spectral Analysis}

The Judd-Ofelt parameters evaluated from least square fitting method are used to predict the radiative properties such as radiative transition probability (A), total transition probability $\left(\mathrm{A}_{\mathrm{T}}\right)$, radiative lifetime $\left(\tau_{R}\right)$, branching ratio $\left(\beta_{\mathrm{R}}\right)$ and stimulated emission cross section $\left(\sigma_{\mathrm{se}}\right)$ [7].

The radiative transition probability A can be expressed from initial $a_{J}$ andfinal $a_{J^{\prime}}$ as,

$$
A=\frac{64 \pi^{4} \lambda_{0}{ }^{3}}{3 h(2 J+1)}\left[\frac{n\left(n^{2}+2\right)^{2}}{9} S_{e d}+n^{3} S_{m d}\right]
$$

Where $\lambda_{0}$ is the peak wavelength of emission band, $S_{\text {ed }}$ and $\mathrm{S}_{\mathrm{md}}$ are the electric and magnetic dipole line strength respectively.

The sum of radiative transition probability from a particular energy level to all the lower levels gives total radiative transition probability $\mathrm{A}_{\mathrm{T}}$.

$$
\mathrm{A}_{\mathrm{T}}=\sum \mathrm{A}
$$

The radiative lifetime $\left(\tau_{R}\right)$ of an excited state is determined using the formula

$$
\tau_{R}=\frac{1}{A_{T}}
$$

The branching ratio $\left(\beta_{\mathrm{R}}\right)$ is another important parameter in understanding the optical potentiality of the prepared glass material which characterizes the possibility of attaining stimulated emission for a specific transition which can be determined by

$$
\beta_{R}=\frac{A}{A_{T}}
$$

The stimulated emission cross section $\left(\sigma_{s e}\right)$ for transition from an initial manifold to final manifold is expressed as

$$
\sigma_{s e}=\frac{\lambda_{p}^{4}}{8 \pi c n^{2} \Delta \lambda_{\text {eff }}} A
$$

Where $\mathrm{n}$ is the refractive index, $\lambda_{\mathrm{p}}$ is the emission peak wavelength and $\Delta \lambda_{\text {eff }}$ the emission effective line width of the transition and is given by

$$
\Delta \lambda_{\text {eff }}=\frac{I}{I_{\max }} \int I(\lambda) d \lambda
$$

Where $I$ is the fluorescence intensity and $I_{\max }$ is the intensity at band maximum.

\subsection{Decay Times and Quantum Efficiencies}

The effective decay time is evaluated using the following expression

$$
\tau_{\text {exp }}=\tau_{\text {eff }}=\frac{\int t I(t) d t}{\int I(t) d t}
$$

Where $\mathrm{I}(\mathrm{t})$ is the emission intensity at time ' $\mathrm{t}$ '. The $\tau_{\exp }$ is expressed using the expression as follows

$$
\frac{1}{\tau_{\exp }}=\frac{1}{\tau_{\text {rad }}}+W_{M P R}+W_{E T}+W_{O H}+\ldots \ldots .
$$

Where $\tau_{\text {rad }}$ is the radiative lifetime and is determined through JO theory, $W_{M P R}$ is the multiphonon relaxation rate, $W_{E T}$ is the rate of energy transfer and $W_{O H}$ is the energy transfer rate between $\mathrm{RE}$ ion and $\mathrm{OH}$ groups present in the glasses.

The non radiative decay rate is determined using the following expression

$$
W_{N R}=\frac{1}{\tau_{\text {exp }}}-\frac{1}{\tau_{\text {rad }}}
$$

The luminescence quantum efficiency $(\eta)$ is defined as the ratio of the number of photons emitted to the number of photons absorbed. In the case of $\mathrm{RE}^{3+}$ ion incorporated systems, it is equal to the ratio of the measured lifetime to the predicted lifetime for the corresponding levels and is given by

$$
\eta=\frac{\tau_{\text {exp }}}{\tau_{\text {rad }}} \times 100 \%
$$

\subsection{Colour Coordinates}

The colour of any light source can be described just by three variables $\mathrm{x}(\lambda), \mathrm{y}(\lambda)$ (colour matching functions), which are dimensionless quantities [8]. The chromaticity coordinates, $\mathrm{x}$ and $\mathrm{y}$ are calculated from the tristimulus values according to the equations:

$$
\begin{aligned}
& x=\frac{X}{X+Y+Z} \\
& y=\frac{Y}{X+Y+Z}
\end{aligned}
$$

Where $\mathrm{X}, \mathrm{Y}$ and $\mathrm{Z}$ are the tristimulus values, which gives the stimulation (i.e. power) for each of the three primary red, green and blue colours needed to match the colour $\mathrm{P}(\lambda)$ (Power spectral density). The locus of all the monochromatic colour coordinates makes the perimeter of CIE (Commission International de I'Eclairage) 1931 chromaticity diagram. All the mono chromatic wavelengths will lie within the area of chromaticity diagram. 


\section{Family of Borate Glasses}

\section{Glass}

It is an amorphous, hard, brittle, super-cooled liquid of infinite viscosity. This non-crystalline solid material is often transparent and has wide spread practical technological \& decorative usage in things such as window panes, tableware and opto-electronics. The inorganic material of glass has low melting point, it can absorb, reflect, and transmit the light.

\section{Borates}

The inorganic oxide glasses like phosphate $\left(\mathrm{PO}_{4}^{4-}\right)$, borate $\left(\mathrm{BO}_{3}{ }^{3-}\right)$, tellurite $\left(\mathrm{TeO}_{3}{ }^{2-}\right)$, germanate $\left(\mathrm{GeO}_{4}{ }^{4-}\right)$, venadate $\left(\mathrm{VO}_{4}{ }^{3-}\right)$ and slicate $\left(\mathrm{SiO}_{4}{ }^{4-}\right)$. It is well known that boron tri oxide $\left(\mathrm{B}_{2} \mathrm{O}_{3}\right)$ is one of the good glass formers and can form glass independently with good transparency, high chemical durability, thermal stability and good rare earth ion solubility [9]. However a glass with boron tri oxide $\left(\mathrm{B}_{2} \mathrm{O}_{3}\right)$ alone will possess high phonon energies $\left(\sim 1300 \mathrm{~cm}^{-1}\right)$ which cannot suppress the non-radiative transitions and hence rare earth ion emissions are strongly reduced. Addition of heavy metal oxides to $\mathrm{B}_{2} \mathrm{O}_{3}$ makes them more stable, moisture resistant, minimize the phonon energies and can give intense fluorescence than the silicates and phosphates [9]. The borate glass environment possess advantageous photonic properties like good transparency, high density, high mechanical stability, good infrared transmission, optimum bandwidth, corrosion resistance is also incost. Borate glasses are commonly used for its wide range of photonic applications such as optical amplifiers, lasers, and have large non-linear refractive index and non-linear absorption coefficients and are promising materials for fiber telecommunication.

\section{Rare Earths/Lanthanides}

Rare earths are a series of chemical elements found in the earth's crust that are vital to many modern technologies, including consumer electronics, computers and networks, communications, clean energy, optical amplifier, advanced transportation, health care, environmental mitigation, national defence, and many others.

Lanthanides are the series of elements from lanthanum (La, $Z=57)$ to lutetium $(\mathrm{Lu}, Z=71)$. The 'rare earth' is the traditional name for the group of elements consisting of the lanthanides together with scandium (Sc) and yttrium (Y).Rare earth-doped glasses are considered to be very important class of photonic materials due to their several inherent advantages over crystalline materials. Doping of glasses with terbium $(\mathrm{Tb})$, europium $(\mathrm{Eu})$, samarium $(\mathrm{Sm})$ or dysprosium (Dy) leads to light emission in the visible range. The beauty of this family of elements is that although the members are very similar from a chemical point of view, each of them has their own specific physical properties including colour, luminescent behavior, nuclear and magnetic properties. In our modern world, lanthanides are becoming very important, especially where light emission is involved. Their very high colour purity is a major advantage of the lanthanides. The trivalent lanthanide ions are known for their sharp electronic transitions in the ultraviolet, visible and near-infrared spectral regions. Excitation and emission spectra of RE ions in glasses are due to $4 \mathrm{f}$ electronic transitions, which are sensitive to the local symmetry, structure of the environment and on phonon energies of the host glass. In order to optimise the efficiency of rare earth doped glasses, the local environment around RE ions has to be changed by changing the glass composition or by changing the concentration of RE ions in a host glass. The ligand fields produced by a host matrix will have considerable influence on the absorption, emission and quantum efficiency of the doped ions. In general a host glass with low phonon energies decreases non radiative relaxation rates and gives high quantum efficiencies [10].

\section{1. $\mathrm{Pr}^{3+}$ and $\mathrm{Er}^{3+}$ Ions Doped in Lithium Cesium Borate Glasses}

The mixed alkali borate glasses were prepared in different mol concentration using melt quenching technique by Y.C. Ratnakaram et al [11]. $\operatorname{Pr}^{3+}$ doped glass fibers are currently viewed as very promising for communication systems based on $1.3 \mu \mathrm{m}$ radiation and integrated optical lasers and amplifiers [12]. They stated among different rare earth ions, erbium is the best suitable ion for up-conversion process because of its energy level structure. The up-conversion process efficiency increases with decrease in non-radiative loss due to multiphonon relaxations. The Judd-Ofelt intensity parameters $\left(\Omega_{2}, \Omega_{4}, \Omega_{6}\right)$ depend on the glass composition and $\Omega_{2}$ parameter increases when second alkali is added to the glass matrix indicating an increase in covalency with addition of second alkali to the binary borate glass. $\Omega_{2}$ parameter is high at $\mathrm{x}=24 \mathrm{~mol} \%$ and low at $\mathrm{x}=$ $8 \mathrm{~mol} \%$ indicating strong and weak covalencies at these compositions in lithium cesium glass matrix. The order of magnitude of Judd-Ofelt parameters is $\Omega_{2}>\Omega_{4}>\Omega_{6}$ for $\mathrm{x}=8,16,20,24 \mathrm{~mol} \%$. For $\mathrm{x}=12 \mathrm{~mol} \%$, the order is $\Omega_{2}>\Omega_{6}>\Omega_{4}$. The intensity of the hypersensitive transition ${ }^{4} I_{15 / 2} \rightarrow{ }^{2} H_{11 / 2}$ decreases with the decrease of $\Omega_{2}$ parameter, which indicates that there is strong interaction between $4 \mathrm{f}$ and $5 \mathrm{~d}$ orbits. The radiative life time $\left(\tau_{R}\right)$ of all the excited states of $\operatorname{Pr}^{3+}$ and $\mathrm{Er}^{3+}$ are more at $\mathrm{x}=$ $8 \mathrm{~mol} \%$. They concluded these glasses are to be a promising candidate for the photonic applications.

\section{2. $\mathrm{Dy}^{3+}$ Ions Doped Strontium Lithium Bismuth Borate Glass (SLBiB)}

Different concentrations of dysprosium doped (SLBiB) glasses were prepared by melt quenching technique by Y.C. Ratnakaram et al [13]. Figure (2) shows the XRD analysis of the prepared glass and which reveals its amorphous nature. 
This type of X-ray diffraction pattern is observable for all glass samples. Raman spectroscopy reveals the coexistence of trigonal $\mathrm{BO}_{3}$ and tetrahedral $\mathrm{BO}_{4}$ units. From the absorption spectra, JO intensity parameters and radiative properties are calculated and the hypersensitivity transition, ${ }^{6} H_{15 / 2} \rightarrow{ }^{6} F_{11 / 2}$ of $\mathrm{Dy}^{3+}$ was discussed based on the magnitude of $\Omega_{2}$ parameter. From the emission spectra, a strong blue emission that corresponds to the transition, ${ }^{4} F_{9 / 2} \rightarrow{ }^{6} H_{15 / 2}$, was observed and it also shows the combination of blue, yellow and red emission band for these glasses. Radiative transition probability (A), effective band widths $\left(\Delta \lambda_{p}\right)$ and stimulated emission cross sections $\left(\sigma_{s e}\right)$ are also calculated and reported for the emission peaks. The white light emission of the glasses were observed and presented.

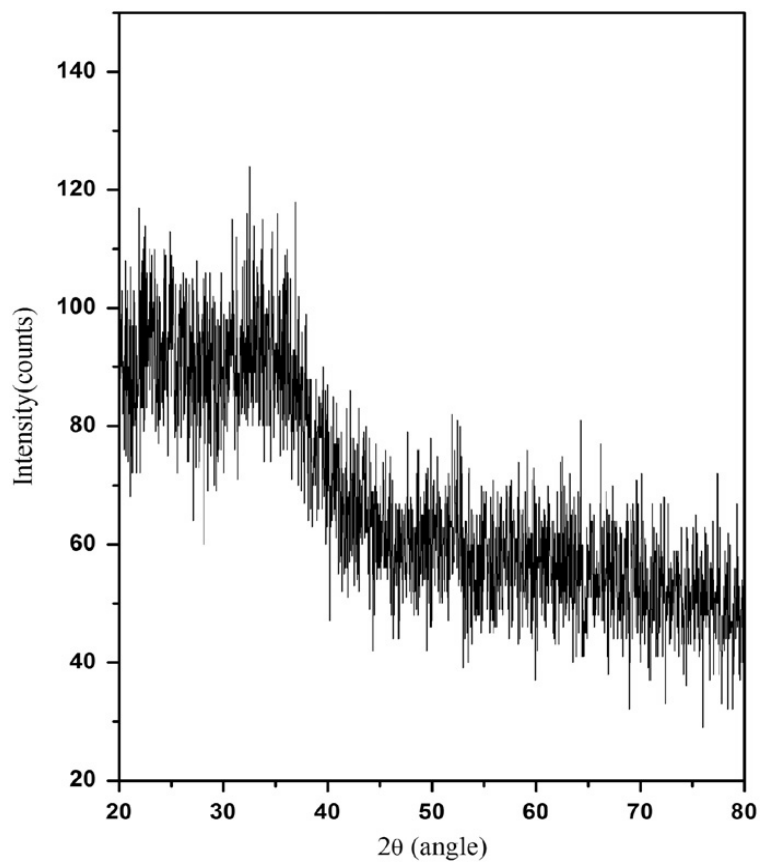

Figure 2. XRD Spectrum of SLBiB glass [13]

\section{3. $\mathrm{Dy}^{3+}$ Ions Doped Zinc Alumino Bismuth Borate Glasses (ZnAlBiB)}

Good optical quality dysprosium doped in zinc alumino bismuth borate (ZnAlBiB) glasses was synthesized in different molar concentrations using melt quenching technique by A. Srinivasa Rao et al [14]. $\mathrm{Dy}^{3+}$ is capable of emitting several interesting wavelengths in its $\mathrm{f}-\mathrm{f}$ transitions that are having potential applications in diversified fields. Among the rare earth ions, $\mathrm{Dy}^{3+}$ is one of the important rare earth ions for the preparation of phosphor material which plays a major role in the preparation of white lighting emitting phosphors. $\mathrm{Dy}^{3+}$ ions have two (yellow and blue) characteristic intense emission bands in the visible region. Physical properties like density (1.80) and refractive index (4.02) and few other parameters were calculated. The XRD analysis shows formation of non crystalline solids and from absorption spectra, the three JO parameters $\Omega_{\lambda}(\lambda=2,4$ and 6) are measured. The intensity of $\mathrm{Dy}^{3+}$ emission spectra increases from 0.5 to $1 \mathrm{~mol} \%$ and beyond $1 \mathrm{~mol} \%$, concentration quenching is observed. The branching ratio and stimulated emission cross-section for two strong transitions such as ${ }^{4} F_{9 / 2} \rightarrow{ }^{4} I_{15 / 2},{ }^{4} F_{9 / 2} \rightarrow{ }^{4} I_{13 / 2}$ are observed in the visible region and CIE chromaticity coordinates were evaluated yellow and blue sharp emissions are observed under $357 \mathrm{~nm}$ excitation which confirms these materials are suitable for white light generation as evidenced in Figure (3).

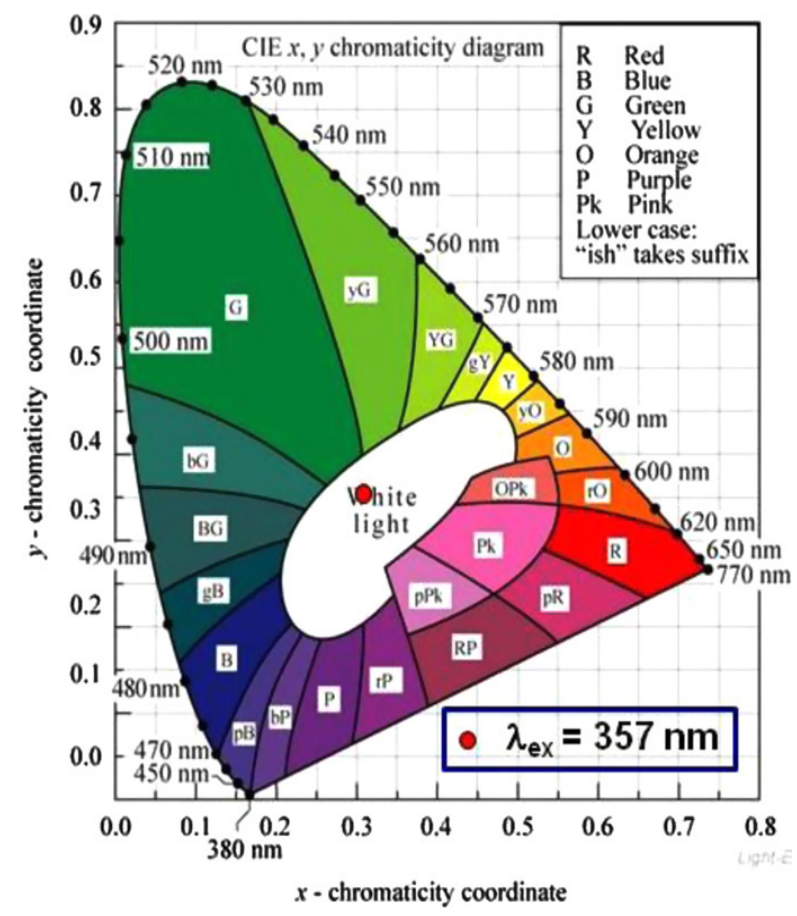

Figure 3. CIE diagram of $\mathrm{ZnAlBiB}$ glass [14]

\section{4. $\mathrm{Nd}^{3+}$ Ions Doped Zinc Alumino Bismuth Borate Glasses (ZnAlBiB)}

Neodymium doped with zinc alumino bismuth borate glasses were prepared in different molar concentrations using conventional melt quenching technique by $\mathrm{A}$. Srinivasa Rao et al [15]. The XRD spectral measurements confirm the glassy nature of the prepared glasses. Physical, absorption and emission properties reveal the lasing potentialities of these glasses. It is also observed that the $\Omega_{2}$, $\Omega_{4}$ and $\Omega_{6}$ are taking maximum values for $1 \mathrm{~mol} \%$ of $\mathrm{Nd}^{3+}$ ion concentration indicating that glass is more symmetric, more covalent and more rigid when compared with other concentrations. Figure (4) shows the emission spectra of the prepared glasses and three prominent transitions ${ }^{4} F_{3 / 2} \rightarrow{ }^{4} I_{9 / 2},{ }^{4} F_{3 / 2} \rightarrow{ }^{4} I_{11 / 2},{ }^{4} F_{3 / 2} \rightarrow{ }^{4} I_{13 / 2}$ are observed.

The intensity of $\mathrm{Nd}^{3+}$ emission spectra increases with increasing concentrations of $\mathrm{Nd}^{3+}$ up to $1 \mathrm{~mol} \%$ and beyond $1 \mathrm{~mol} \%$ concentration quenching is observed. Figure (5) shows the energy level diagram of $\mathrm{Nd}^{3+}$ ion. High stimulated emission cross section and branching ratios of these glasses have potential for infrared lasers. 


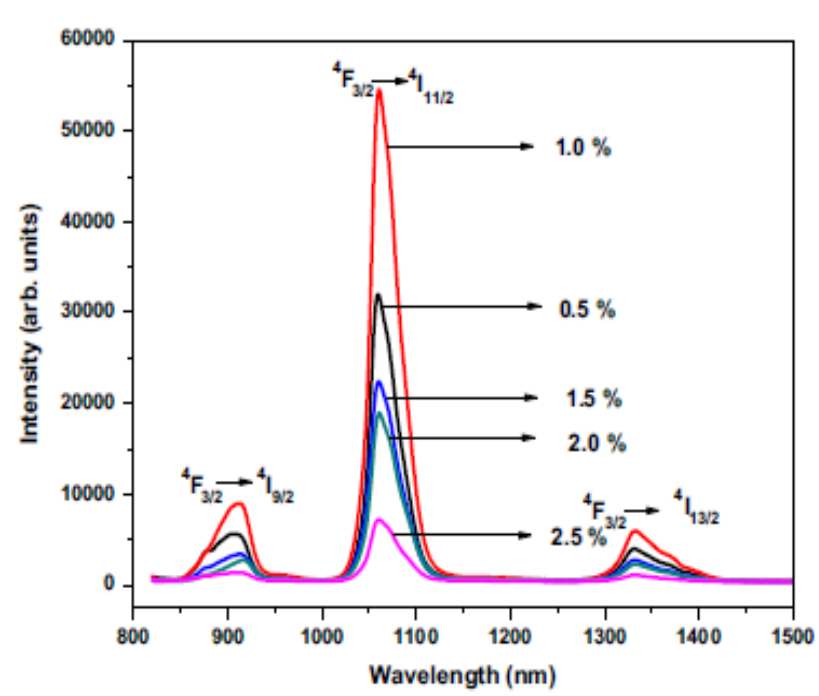

Figure 4. Emission Spectra of ZnAlBiB glass[15]

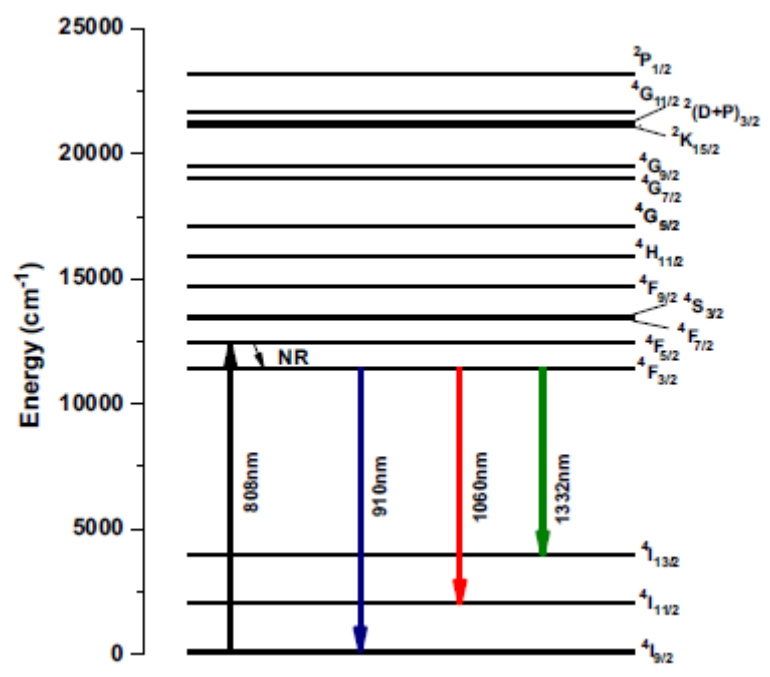

Figure 5. Energy level diagram of ZnAlBiB glass[15]

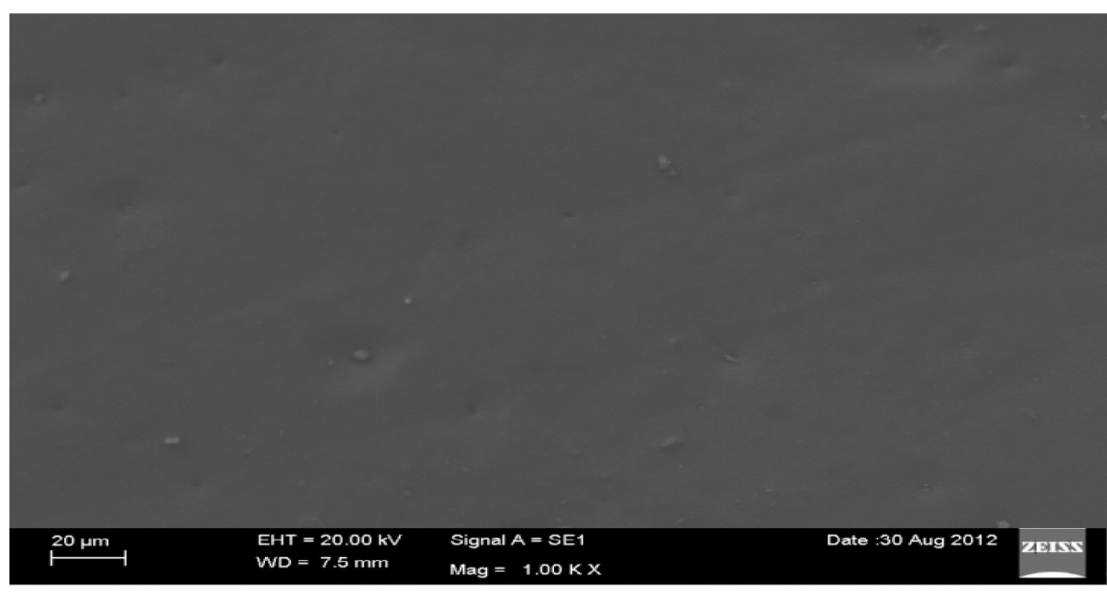

(a)

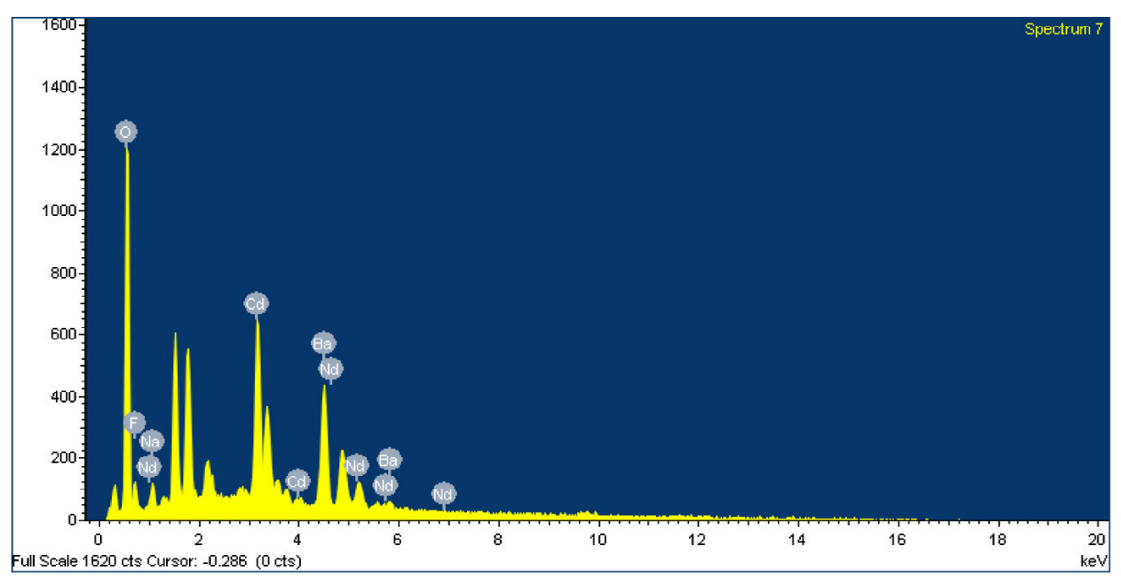

(b)

Figure 6a. SEM image and $6 \mathrm{~b}$ EDS Spectra of LFB glass [16]

\section{5. $\mathrm{Nd}^{3+}$ Ions Doped Lithium Fluoro Borate Glasses (LFB)}

Different modifier oxides based neodymium doped (LFB) glasses were synthesized by melt quenching technique by
Y.C. Ratnakaram et al [16]. The spectra exhibits a broad scattering which represents a typical long range structural disorder and confirms the amorphous nature of the glass. Figure (6)a and (6)b shows the SEM image and EDS spectrum of the glass. From the SEM image it was found that 
clear smooth glassy surface with no formation of clusters were observed. The EDS spectrum reveals all the elements that are present in the investigated glass samples in relative amounts. Optical absorption and photoluminescence spectra were recorded. JO intensity parameters were calculated and are used to explain some radiative properties. The emission transition, ${ }^{4} F_{3 / 2} \rightarrow{ }^{4} I_{11 / 2}$ have large stimulated emission cross section and branching ratio values. Finally, it is concluded that the ${ }^{4} F_{3 / 2}$ level gives the highest quantum efficiency for all these glasses. It indicates the efficient laser emission at $1.06 \mu \mathrm{m}$ and acts as a good laser active media for laser applications.

\section{6. $\mathrm{Eu}^{3+}$ Ions Doped Telluro Fluoro Borate Glasses (TFBO)}

The glasses of europium doped telluro fluoro borate (TFBO) were prepared in different molar concentrations using melt quenching technique by K.Marimuthu et al [17]. The optical properties of absorption, excitation and luminescence were studied. Physical properties like density (2.97), refractive index (1.61) and some other parameters were calculated. Nephelauxetic ratio $\left(\beta_{R}\right)$ and bonding parameters $(\delta)$ were calculated from absorption spectra and reveals that nature of bonding between the $\mathrm{Eu}^{3+}$ ion and its surrounding ligands. The optical band gap and Urbach energy also calculated from absorption spectra to study the electronic band structure and it's observed that, the band gap values vary inversely with the Urbach energy values. Judd-Ofelt intensity parameters were calculated from the emission spectra provides information about all the radiative properties. From emission spectra two emission transition ${ }^{5} D_{0} \rightarrow{ }^{7} F_{2},{ }^{5} D_{0} \rightarrow{ }^{7} F_{1}$ has been calculated and determine the degree of asymmetry around the $\mathrm{Eu}^{3+}$ ion site and to identify the strength of the covalence of $\mathrm{Eu}^{3+}$ ion with its surrounding ligands. Figure (7) shows CIE chromaticity colour coordinates of these glasses.

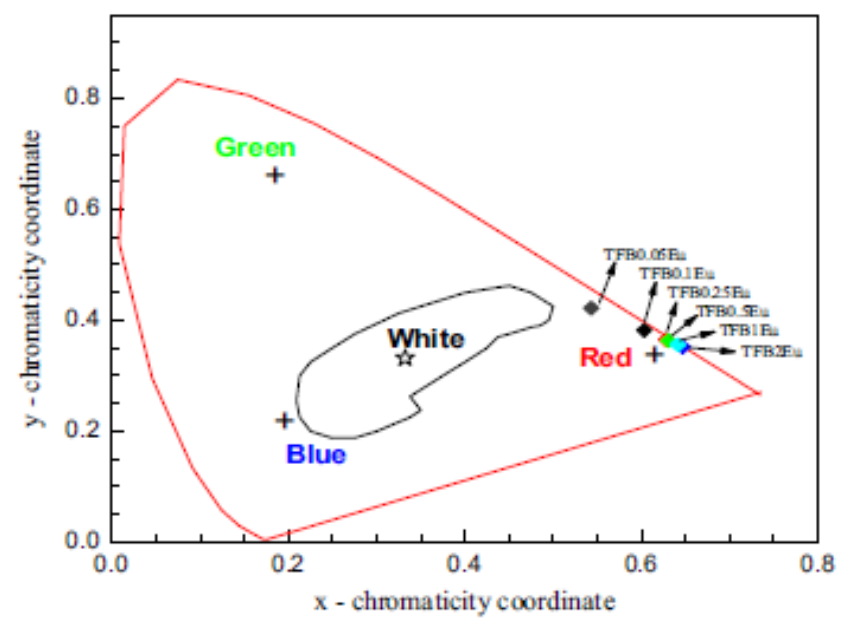

Figure 7. CIE Diagram of TFBO glass [17]

\section{7. $\mathrm{Ho}^{3+}$ Ion Doped Lithium Fluoro Borate Glasses (LFB)}

Holmium ions have wide transparency in the ultraviolet to infrared region. For this purpose they prepared holmium doped (LFB) glasses using conventional melt quenching technique by Y.C. Ratnakaram et al [18]. Structural analysis of XRD, SEM reveals glassy nature for these glasses. FT-IR shows good bond formation and JO intensity parameters were calculated and used explain radiative properties. Figure (8) shows the Raman spectra of the prepared glasses with different modifier oxides. Raman band observed in the low frequency $\left(185 \mathrm{~cm}^{-1}\right)$ is associated to the symmetric M-O stretching in the $\mathrm{MO}_{4}$ pyramid configuration (where $\mathrm{M}=\mathrm{Mg}$, $\mathrm{Ca}, \mathrm{Cd}, \mathrm{Pb})$. The band in $\left(452 \mathrm{~cm}^{-1}\right)$ could be attributed B-O-B bending vibrations. The strong band at $\left(674 \mathrm{~cm}^{-1}\right)$ and $\left(1334 \mathrm{~cm}^{-1}\right)$ are attributed to the meta borate groups containing non-bridging oxygen and stretching vibrations of $\mathrm{B}^{-} \mathrm{O}^{-}$in different borate groups. Finally cadmium glass matrix has larger stimulated emission cross section and branching ratio which indicates that this is a good lasing material at this wavelength and highly useful for laser excitation.

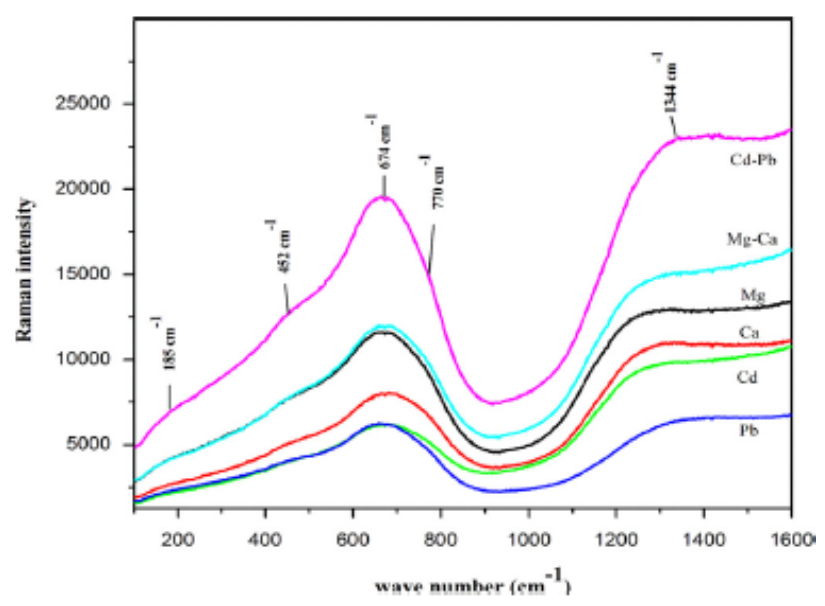

Figure 8. FTIR spectra of LFB glass [18]

\section{8. $\mathrm{Sm}^{3+}$ Ions Doped Zinc Alumino Bismuth Borate Glasses (ZnAlBiB)}

Luminescent material of zinc alumino bismuth borate Glasses was prepared in different mol concentration using the melt quenching technique by A. Srinivasa Rao et al [19]. The grown glasses were subjected to X-ray diffraction analysis and it shows amorphous structure. The density (3.54), refractive index (1.81) and some other physical parameters are calculated and reported. The fluorescence intensity of $\mathrm{Sm}^{3+}$ ion depends on the concentration of RE ions as well as on the glass network. The covalent character of $\mathrm{Sm}^{3+}$ ions with its surrounding ligands has been confirmed through optical spectra. The absorption and emission spectra of these glasses were recorded and analyzed using Judd-Ofelt theory. The reasonably small $\mathrm{rms}$ deviations observed between the measured and calculated oscillator. From this evaluated JO parameters, the radiative properties such as transition probability, radiative lifetimes, branching ratios and integrated absorption cross sections were calculated. The emission spectra recorded for all the glasses 
show two intense emissions one at orange region $\left({ }^{4} G_{5 / 2} \rightarrow{ }^{6} H_{7 / 2}\right)$ and the other one at red region $\left({ }^{4} G_{5 / 2} \rightarrow{ }^{6} H_{9 / 2}\right)$. Finally it is concluded and based on the physical and optical properties strong visible emissions, large stimulated emission cross sections, high branching ratios and good quantum efficiencies are possible, and this lasing material is suitable for the development of laser and photonic devices operating in the visible region.

\section{9. $\mathrm{Er}^{3+}$ Ions Doped Barium Telluro Fluoro Borate Glasses (BTFB)}

The glasses of erbium doped barium tellurofluoroborate were prepared in different molar concentrations using conventional melt quenching technique by K.Marimuthu et al [20]. The density (5.21), refractive index (1.71) and few other physical parameters like polaran radius, field strength, molar refractivity, dielectric constant, reflection losses and electronic polarizability were calculated. The X-ray diffraction analysis reveals that the prepared glasses are in amorphous nature. The infrared spectroscopy is shows that particular functional groups are present. The absorption intensity is found to increase as the erbium ion concentration in the glass matrix increases. The Nephelauxetic ratio $(\beta)$ and bonding parameter $(\delta)$ studies indicate that the ionicity of the Er-O bond decreases as the concentration of RE ion in the host matrix increases. The product of FWHM and stimulated emission cross section of $0.5 \mathrm{~mol} \%$ of the glass has the maximum value of 134.87. Finally these glasses are suggested to be a promising candidate for the fabrication of optical devices and photonic applications.

\section{Conclusions}

The present review work presents a consolidated overview on parameters related to glasses for optical applications, mainly for realizing white light, and an analysis of potential borate glasses till now.

\section{REFERENCES}

[1] Sybil P. Parker, Spectroscopy Source Book (1989).

[2] Ho Li Ngee, Tomoki Hatsumori, Kenji Toda, Mineo Sato, Kazuyoshi Uematsu, "Synthesis of phosphate phosphor for a white LED," Physics Procedia 2 (2009) 171-183.

[3] J. Reichman, Handbook of Optical Filters for Fluorescence Microscopy (Chroma Technology, Brattleboro, 2010).

[4] K. N. Shinde, S. J. Dhoble, H. C. Swart, K. Park, Basic Mechanics of Photoluminescence- "Phosphate phosphors for solid-state lighting", Springer.
[5] W. T. Carnall, P.R. Fields, K. Rajnak, J. Chem. Phys. 49 (1968) 4424-4442.

[6] R. Reisfeld, L. Boehm, J. Slid state Chem. 4 (1972) 417.

[7] V. Mehta, G. Aka, A. L. Dewar, A. Mansingh, Opt. Mater. 12 (1999) 53-63.

[8] E. F Red Schubert, Light Emitting Diodes, vol. 292, second edition., Cambridge University, 2006, (P.300).

[9] H. Yamanchi, Y. Onishi, Opt. Mater. 27 (2005) 679-690.

[10] Y. G. Choi. J. Heo, Journal of Non-Crystalline Solids 217 (1997) 199.

[11] Y. C. Rathnakaram, A. Vijaya Kumar, R. P. S. Chakradhar, "Optical absorption and emission properties of $\mathrm{Pr}^{3+}$ and $\mathrm{Er}^{3+}$ in lithium cesium mixed alkali borate glasses," Journal of Luminescence 118 (2006) 227-237.

[12] K. Wei, D.P. Machewirth, J. Wenzel, E. Snitzer, G. H. Sigel Jr., J. Non Cryst. Solids 182 (1995) 1842.

[13] D. Rajesh, Y. C. Ratnakaram, M. Seshadri, A. Balakrishna, T. Satya Krishna, "Structural and luminescence properties of $\mathrm{Dy}^{3+}$ ion in Strontium Lithium Bismuth Borate Glasses," Journal of Luminescence 132 (2012) 841-849.

[14] K. Swapna, Sk. Mahamuda, A. Srinivasa Rao, M. Jayasimhadri, T. Sasikala, L.Rama Moorthy, "Optical absorption and luminescence characteristic of $\mathrm{Dy}^{3+}$ ions doped Zinc Alumino Bismuth Borate Glasses for lasing materials and white LEDs," Journal of Luminescence 139 (2013) 119-124.

[15] Sk. Mahamuda, K. Swapna, A. Srinivasa Rao, M. Jayasimhadri, T.Sasikala, K. Pavani, L. Rama moorthy, "Spectroscopic properties and luminescence behaviour of $\mathrm{Nd}^{3+}$ ions doped Zinc Alumino Bismuth Borate Glasses," Journal of Physics and Chemistry of Solids 74 (2013) 1308-1315.

[16] A. Balakrishna, D. Rajesh, Y.C. Ratnakaram, "Structural and optical properties of $\mathrm{Nd}^{3+}$ ions doped Lithium Fluoro Borate Glass with relevant modifier oxides," Optical Materials 35 (2013) 2670-2676.

[17] R. VijayaKumar, K.Maheswaran, V.Sudarsan, K. Marimuthu, "Concentration dependent luminescence studies on $\mathrm{Eu}^{3+}$ doped telluro fluoroborate Glasses," Journal of Luminescence 154 (2014) 160-167.

[18] A. Balakrishna, Y.C. Ratnakaram, D. Rajesh, "Spectroscopic analysis of $\mathrm{Ho}^{3+}$ transitions in different modifier oxide based lithium fluoro borate Glasses," Physica B 450 (2014) 58-66.

[19] A. Srinivas Rao, T. Saaikala, L.Rama Moorthy, K. Swapna, SK. Mahamuda, "Visible luminescence characteristic of $\mathrm{Sm}^{3+}$ ions doped Zinc Alumino Bismuth Borate Glasses," Journal of Luminescence 146 (2014) 288-294.

[20] K.Annapoorani, K. Maheswaran, S. Arunkumar, N. Suriya Murthy, K.Marimuthu, "Structural and luminescence behaviour of Er3+ ions doped Barium tellurofluoroborate glasses," Spectrochimica Acta Part A: Molecular and Biomolecular Spectroscopy 135 (2015) 1090-1098. 